\title{
Measurement and Feature Analysis of Plantar Pressure Center in Athletes under Different Exercise Modes
}

\author{
Jie Yang \\ Physical Education Department, Guizhou University of Commerce, Guiyang 550014, China
}

Corresponding Author Email: 201510473@gzcc.edu.cn

https://doi.org/10.18280/i2m.190511

Received: 2 June 2020

Accepted: 17 September 2020

\section{Keywords:}

plantar pressure center (PPC), gait analysis, trajectory, training, exercise mode

\begin{abstract}
Walking and running, two essential exercises in daily training for athletes, are major causes to foot injuries. The plantar pressure center (PPC) can accurately reflect the gait process, and effectively measure the function of foot. This paper measures the PPC trajectories of a total of 45 athletes during the support period of walking and running, and analyzes their regularities under different exercise modes. The time percentage of PPC trajectory and foot progression angle were selected as the main observation indices. The support period under each exercise mode was divided into four phases: the initial contact phase (ICP), the forefoot contact phase (FFCP), the foot flat phase (FFP), and the forefoot push off phase (FFPOP). The statistical analysis shows that: Under the running mode, the time of the heel landing on the ground and the relative load on the heel decrease with the growing speed. Under the same exercise mode, the left and right feet differ slightly in the PPC trajectory; the foot progression angle peaks in FFPOP and minimizes in FFP. In ICP and FFPOP, the foot progression angle under running is smaller than that under walking; in FFP and FFCP, the foot progression angle under running is larger than that under walking. The research results provide the scientific basis for the reasonable arrangement of athlete training.
\end{abstract}

\section{INTRODUCTION}

Walking and running are two indispensable exercises in daily training for athletes. These exercises can greatly improve the aerobic capacity $[1,2]$, but brings a potential risk of injury $[3,4]$. During the exercises, the foot is particularly prone to injury, because the foot force is 4.6 times of body weight [5].

Foot absorbs much of the shock in the process of the contact with the ground. It can adapt to irregular surface, and generate the power to move forward. The foot structure and function, as well as the movement of body posture, can be reflected accurately by the distribution of plantar pressure. By measuring and analyzing plantar pressure, it is possible to obtain the mechanical and functional parameters of the body in various postures and motion states.

The point that the instantaneous ground reaction force acts on the sole is called the plantar pressure center (PPC), which has been widely used to describe the complex dynamic functions of the foot and the sole interface in the gait process [6]. The PPC trajectory is composed of a series of PPC coordinates, as it moves from the hind foot to the forefoot. The space-time features of the PPC trajectories shed important light on the structure and dynamic functions of the foot. Previous studies have shown a significant correlation between the PPC trajectory and age $[7,8]$. The trajectories parameters have been used to calculate the sole size, body mass, and body damage $[9,10]$.

The displacement of the PPC mirrors the inner-lateral and forward-backward movements of the foot, providing an important metric of foot functions. During forward movement, the PPC displacement reflects the dynamic action of the foot.
The central trajectories of the second metatarsal bone and the outside of the foot can be observed when the forefoot pedals on the ground [11]. The PPC velocities of young and middleaged people have three peaks in the range of $22-27 \mathrm{~cm} / \mathrm{s}$ and $38 \mathrm{~cm} / \mathrm{s}$ during walking [12]. Willems et al. identified the PPC displacement as a risk factor for sprain and exercise-related leg pain $[13,14]$. The PPC has been considered to study the gates of diabetes and stroke patients $[15,16]$.

This paper explores the PPC trajectories during the support period under walking and running modes, and analyzes the features and regularities of the PPC trajectory under different exercise modes, revealing the influence of exercise mode on the PPC trajectory. In addition, athlete samples were collected to verify the analysis results. The research findings provide scientific basis for clinical diagnosis and rehabilitation of foot injuries induced by sports training.

\section{METHODOLOGY}

Our tests were conducted in a sports biomechanics lab from July to September, 2020, in China. The main instruments include a high-frequency plantar pressure test board (length: $2 \mathrm{~m}$; acquisition frequency: $126 \mathrm{~Hz}$; sensor density: $4 \mathrm{~N} / \mathrm{cm}^{2}$; measuring range: $1-60 \mathrm{~N} / \mathrm{cm}^{2}$; minimum resolution: $35 \mathrm{~g}$; consistency: $\pm 25 \mathrm{~g}$ ), and a three-dimensional (3D) foot scanner (effective area: $350 \mathrm{~mm} \times 150 \mathrm{~mm} \times 160 \mathrm{~mm}$; accuracy: $\pm 1 \mathrm{~mm}$ ).

A total of 45 young male athletes were selected. All of them are able to walk normally, and free of any abnormalities that detriment normal walking, namely, cardiovascular or neuromuscular diseases, foot deformities, and ankle injuries. 
The basic information of the subjects is shown in Table 1.

Table 1. The basic information of the athletes

\begin{tabular}{cc}
\hline Items & Value \\
\hline Total number & 45 \\
Age & $22.5 \pm 2.7$ \\
Height $(\mathrm{cm})$ & $177.2 \pm 6.7$ \\
Weight $(\mathrm{kg})$ & $73.6 \pm 10.2$ \\
\hline
\end{tabular}

Furthermore, the selected subjects all have normal arch shapes. Their foot arch indices fell between 0.21 and 0.26 , excluding high and low arches.

Following self-control method, the gait analysis and testing won informed consent from every subject. Firstly, the basic data (i.e. age, weight, and height), and foot measurements were collected. The pressure test board was plaid on a smooth ground, with two ends covered with extended runway. Then, each subject took off his shoes and shoes, and walked or ran naturally across the pressure test board. The plantar pressure was measured three times under normal walking and normal running, respectively. Based on the measurements, a complete plantar pressure gait analysis diagram was plotted by the plantar pressure analytic system.

Next, a PPC coordinate system was established with the yaxis being the straight line from the hind foot to the upper side of the second metatarsal bone of the forefoot, and the x-axis being perpendicular to the y-axis. The feet of the subjects were normalized. A single average image was registered to the specified template. The PPC trajectories were extracted from all subjects for statistical analysis. In order to avoid deviations related to template selection, the data were re-registered to the obtained average image.

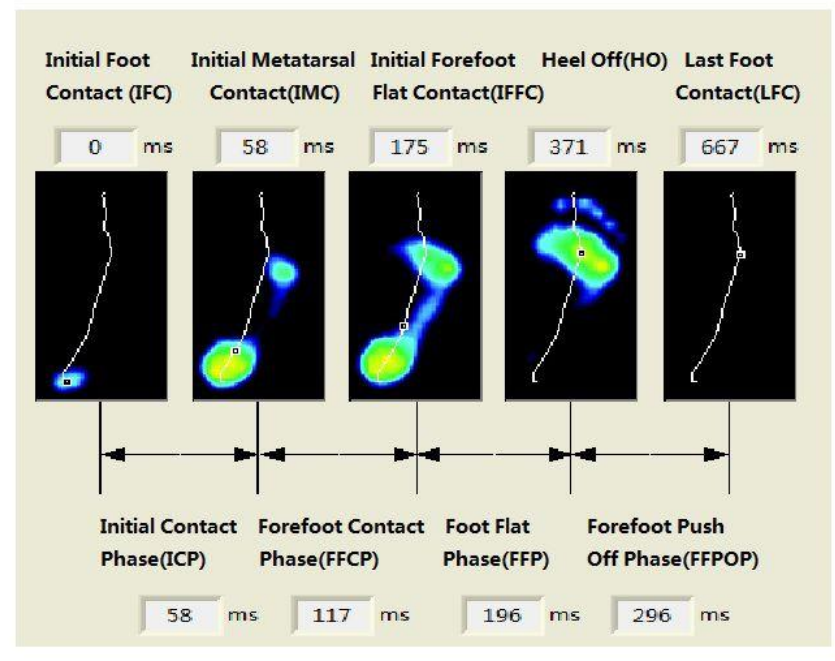

Figure 1. The schematic diagram of four phases

As shown in Figure 1, each PPC trajectory can be divided into four phases: the initial contact phase (ICP) from the first contact with the pressure test board to the first metatarsal touching the board; the forefoot contact phase (FFCP) from the end of ICP to all metatarsal head areas touching the board; the foot flat phase (FFP) from the end of FFCP to the heel just off the board; the forefoot push off phase (FFPOP) from the end of FFP to the entire foot off the board. Figure 2 shows the flow chart of our tests.

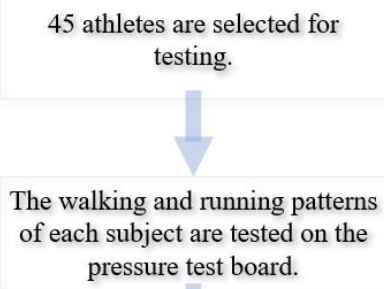

Figure 2. The flow chart of our tests

As shown in Figure 2, the time percentage of PPC trajectory and foot progression angle are the main observation indices. The time percentage of PPC trajectory refers to the normalized PPC trajectory with different time lengths. In each phase, the time percentage equals the ratio of the phase length to the support period. The foot progression angle refers to the angle between the forward direction in the support period and the line between the starting and ending points in each phase.

The collected data were processed on SPSS statistical software, and analyzed on the software embedded in the measuring instruments. The statistical results were expressed by $x \pm s$. Paired sample t-test was conducted, with $\mathrm{P}<0.05$ as the significance level.

\section{RESULTS ANALYSIS}

To ensure the reliability of measurement, PPC displacement was recorded in $\mathrm{x}$ and $\mathrm{y}$ coordinates. For data consistency, the reliability of the $\mathrm{x}$ and $\mathrm{y}$ coordinates of the PPC was evaluated by the intra-class correlation coefficient (ICC 2, 1), using the confidence interval of $95 \%$. If the ICC is greater than 0.75 , the reliability is highly significant; if the ICC is between 0.75 and 0.40 , the reliability is slightly significant; if the ICC is smaller than 0.40 , the reliability is not significant.

The evaluation results show that the ICC values of $\mathrm{x}$ coordinate of PPC displacement were 0.74 and 0.74 under walking and running modes, respectively; those of $y$ coordinate of PPC displacement were 0.97 and 0.96 under walking and running modes, respectively. Overall, the PPC displacements under both exercise modes have good reliability. Table 2 presents the t-test results on the time percentages of PPC trajectory under walking and running modes.

For the left foot, there are significant differences $(\mathrm{P}<0.05)$ in the time percentages of ICP, FFCP, and FFP in PPC trajectory between walking and running modes, but no significant difference $(\mathrm{P}>0.05)$ in that of FFPOP. For the right foot, there are significant differences $(\mathrm{P}<0.05)$ in the time percentages of all four phases in PPC trajectory between walking and running modes. Table 3 shows the t-test results on the time percentages of PPC trajectory under the same mode. 
Table 2. The t-test results on the time percentages of PPC trajectory under walking and running modes

\begin{tabular}{ccccccc}
\hline \multirow{2}{*}{ Phase } & \multicolumn{3}{c}{ Left foot } & & \multicolumn{3}{c}{ Right foot } \\
\cline { 2 - 7 } & Walking & Running & P value & Walking & Running & P value \\
\hline ICP & $7.26 \pm 1.79$ & $5.06 \pm 1.18$ & 0.000 & $7.23 \pm 1.82$ & $4.99 \pm 1.19$ & 0.000 \\
FFCP & $8.39 \pm 3.41$ & $5.76 \pm 2.08$ & 0.005 & $8.38 \pm 3.39$ & $5.81 \pm 2.03$ & 0.011 \\
FFP & $31.92 \pm 5.61$ & $36.99 \pm 6.21$ & 0.002 & $31.27 \pm 5.58$ & $37.12 \pm 6.16$ & 0.000 \\
FFPOP & $52.18 \pm 8.29$ & $50.90 \pm 5.72$ & 0.995 & $51.92 \pm 8.16$ & $51.02 \pm 5.80$ & 0.018 \\
\hline
\end{tabular}

Table 3. The t-test results on the time percentages of PPC trajectory under the same mode

\begin{tabular}{ccccccc}
\hline \multirow{2}{*}{ Phase } & \multicolumn{3}{c}{ Walking } & & & Running \\
\cline { 2 - 7 } & Left foot & Right foot & P value & Left foot & Right foot & P value \\
\hline ICP & $7.26 \pm 1.79$ & $7.23 \pm 1.82$ & 0.745 & $5.06 \pm 1.18$ & $4.99 \pm 1.19$ & 0.637 \\
FFCP & $8.39 \pm 3.41$ & $8.38 \pm 3.39$ & 0.988 & $5.76 \pm 2.08$ & $5.81 \pm 2.03$ & 0.899 \\
FFP & $31.92 \pm 5.61$ & $31.27 \pm 5.58$ & 0.096 & $36.99 \pm 6.21$ & $37.12 \pm 6.16$ & 0.516 \\
FFPOP & $52.18 \pm 8.29$ & $51.92 \pm 8.16$ & 0.052 & $50.90 \pm 5.72$ & $51.02 \pm 5.80$ & 0.633 \\
\hline
\end{tabular}

Under walking mode, there are significant differences $(\mathrm{P}<$ $0.05)$ in the time percentages of the four phases. Under running mode, there is no significant difference $(\mathrm{P}>0.05)$ in the time percentages of the four phases.

The time percentages of the four phases and the corresponding foot progression angles are recorded in Figure 3 , where the $y$ axis is the longitudinal axis of the foot, i.e. the line from the mid-heel (the first pressure center) to the front foot (above the second metatarsal), the $\mathrm{x}$ axis is perpendicular to the y axis, the left subgraph is the PPC trajectory under running mode, and the right subgraph is the PPC trajectory under walking mode.

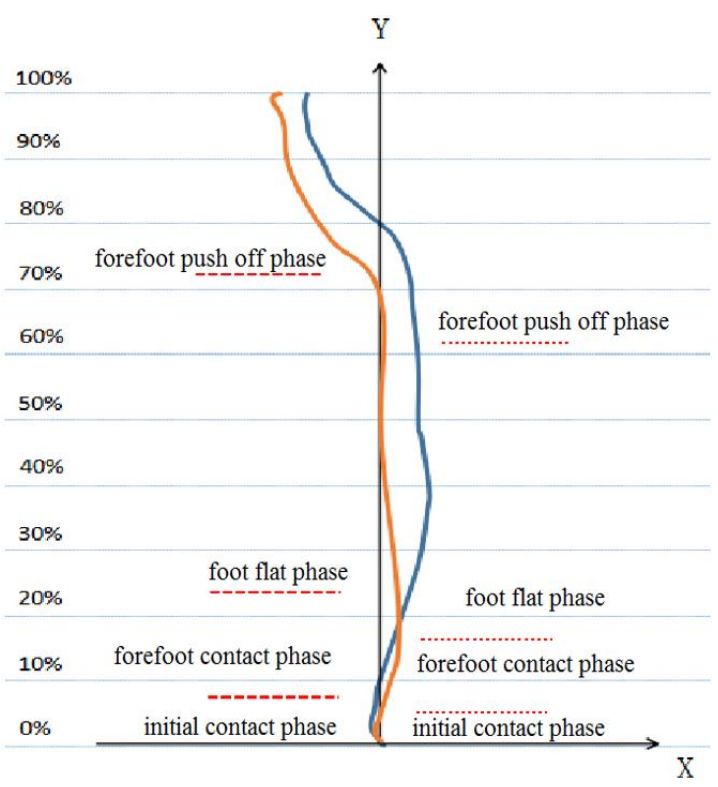

Figure 3. The time percentages of the four phases and the corresponding foot progression angles

As shown in Figure 3, the PPC trajectory under the running mode was closer to the centerline of the foot than that under the walking mode. The foot progression angle of each phase was derived from the corresponding PPC displacement. On the $\mathrm{x}$ axis, the PPC shifted inward in the negative direction, and skewed outward in the positive direction. Taking the absolute values of $\mathrm{x}$ coordinates in walking and running modes, the coordinates $(\mathrm{x}, \mathrm{y})$ on both sides of the $\mathrm{x}$ axis were obtained. The left and right coordinates $(x, y)$ were connected with their starting and end points, thereby producing the foot progression angle in each phase. Comparatively, the PPC trajectory under running mode was more inward than that under walking mode. Figure 4 and Table 4 present the foot progression angles in each phase under the two exercise modes.

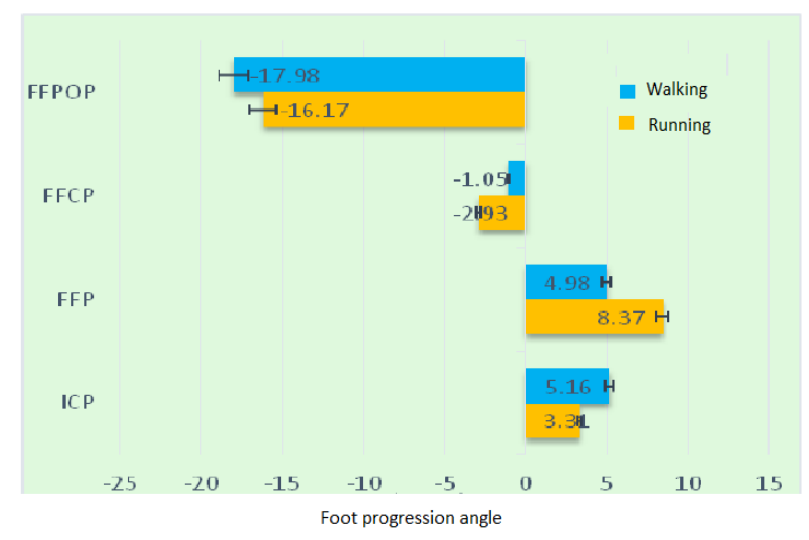

Figure 4. The foot progression angles in each phase under the two exercise modes

Table 4. The foot progression angles in each phase under the two exercise modes

\begin{tabular}{cccc}
\hline Phase & Walking & Running & P value \\
\hline ICP & $5.18 \pm 1.62$ & $3.29 \pm 1.41$ & 0.005 \\
FFCP & $4.97 \pm 1.81$ & $8.36 \pm 2.39$ & 0.004 \\
FFP & $-1.06 \pm 0.91$ & $-2.88 \pm 1.16$ & 0.006 \\
FFPOP & $-17.89 \pm 5.96$ & $-17.06 \pm 3.99$ & 0.050 \\
\hline
\end{tabular}

As shown in Table 4 and Figure 4, the four phases differed significantly in foot progression angle $(\mathrm{P}<0.05)$ : the foot progression angle under walking mode was greater than that under running mode in the ICP and FFPOP, and smaller than the latter in FFCP and FFP; the foot progression angles under both modes were positive in the ICP and FFCP, and negative in the FFP and FFPOP.

\section{DISCUSSION}

Under the walking mode, each athlete lands firstly on the heel. Thus, the PPC trajectory in the ICP exhibits a C-shape. When the foot leaves the ground, the curve of the forefoot leaving the ground follows the dorsal curvature of metatarsalphalangeal, similar to the $\mathrm{C}$-shaped trajectory.

Comparing the walking and running modes, it can be seen 
that the foot progression angles in PPC trajectory vary significantly with exercise modes. The PPC trajectory under the running mode has a greater foot progression angle than that under the walking mode. During the support period, the PPC trajectory can be split into the ICP, FFCP, FFP, and FFPOP. In the process of running, the foot utilizes more muscle fibers to pedal and pick the ground than it does under the walking mode. Besides, the muscles contract more forcefully in running mode than in walking mode. The more stiff of the foot, the greater the kicking off the ground, and the larger the reaction force from the ground.

The ICP has a relatively small foot progression angle. This is consistent with the PPC displacement, and the anatomically limited moving range of the subtalar joint. In the ankle joint, there is an active neuromuscular control mechanism that enhances the movement of the medial lateral muscles. The running mode has a greater foot progression angle, and a much smaller lateral displacement than walking mode. In addition, the metatarsal-phalangeal dorsal curvature in running is smaller than that in walking.

At the beginning of landing, the heel is slightly pronated, and its outer part is in contact with the ground. Fast foot progression helps absorb the impact of body weight. The responses of ankle and subtalar joints affect ankle plantar flexion and subtalar valgus. The supination of subtalar joint valgus makes the transverse ankle joint more flexible, improving the impact absorbance. Our research results show significant differences between left and right feet in the time percentage of ICP, FFCP, and FFP in PPC trajectory under walking and running modes, yet the two feet differed insignificantly in the time percentages of FFPOP. In the ICP, the time percentage and foot progression angle under running are smaller than those under walking. During running, the center of gravity of the body is more inclined forward, the heel progression in faster, and the foot progression angle is smaller than that under the walking mode. These patterns can quickly relieve the impact on foot, and speed up the transition to the next phase. During running, the relative load of heel decreases with the growing speed, although the overall load on the hell of a running athlete is greater than that on a walking athlete.

After the ICP, the peak velocity of the PPC moves rapidly forward, followed by fast ankle flexion and pronation. Meanwhile, the lateral displacement of PPC transmits pressure to the lateral foot. The time percentage of this phase under walking is greater than that under running. The two exercise modes differed significantly in the time percentage. However, walking mode has a smaller foot progression angle than the running angle. Compared with that in ICP, the PPC trajectory in FFCP varied very significantly. The fast pronation of the foot leads to two different peak velocities. In addition, the ankle joint, the leg, and the knee have a mechanical coupling. In this phase, the instantaneous medial load is consistent with the rapid initial rotation of the tibia. The PPC displacement in ICP and FFCP may be an important cause of foot load and lower limb injury.

In the FFP, the foot supports the entire body through ankle dorsiflexion and heel elevation. The PPC displacements under walking and running are smaller than those in the previous phase. The main purpose of the displacements is to keep the body stable and absorb shocks. Compared with the walking mode, the running mode has a short FFP with fast PPC movement, large time percentage, and fluctuating foot progression angle. The large PPC displacement is attributable to the inversion of the subtalar joint to fix the transverse talus joint, which leads to a rigid structure of the plantar aponeurosis as the body moves on a weight-bearing limb. According to the stiffness of foot-ground contact surface from hard to soft lower extremities, the stiffness of lower extremities increases with lateral displacement, and the PPC trajectories decreases with the growing internal displacement. The features of running movement indicate that a small landing distance can increase the angle of pedal, reduce resistance, and improve running speed.

In the FFPOP, the main movements include extension of the knee and plantar, plus the dorsal extension of ankle. Under both exercise modes, the PPC trajectory exhibited very significant displacement, suggesting that medial metatarsal and toe areas are pushed off the ground. Although the two modes have an unobvious difference in time percentage, the FFPOP has the largest foot progression angle among the four phases; this angle is larger under walking than under running. Since running brings an impact force three times that indued by walking, more internal rotation might be caused by this intense training mode. The walking mode mainly affects the medial part of the second metatarsal, while the running mode mainly affects the area above the medial part of the big toe and metatarsal. These results are consistent with the previous findings $[17,18]$.

In the four phases under the same exercise mode, there is no significant difference between left and right feet in the time percentage. In fact, the two feet have similar time touching the ground under walking and running modes. This is conducive to maintaining the balance of the body and the coordination and stability of the movement. Under the same mode, the PPC trajectory is highly similar through different phases. The high similarity may be related to the consistency of exercise mode. In either mode, the gait inconsistency caused by foot injury or nerve injury will inevitably tilt the balance of the PPC trajectory, while reflecting the therapeutic effect or improvement degree of sports injury.

\section{CONCLUSIONS}

This paper measures the PPC trajectories of a total of 45 athletes during the support period of walking and running, and analyzes the features of these trajectories under different exercise modes, aiming to disclose the influence of walking and running on the foot pressure of athletes. The main conclusions are as follows:

(1) Walking and running modes have many differences in the four phases. Under the running mode, the time of the heel landing on the ground and the relative load on the heel decrease with the growing speed.

(2) Under the same exercise mode, the left and right feet differ slightly in the PPC trajectory. Different exercise modes can be differentiated by the PPC trajectory. The running mode has greater internal PPC displacement than the walking mode.

(3) Under either walking or running mode, the foot progression angle peaks in FFPOP and minimizes in FFP. In the first two phases, the foot rotates outward; in the last two phases, the foot rotates outward.

(4) In ICP and FFPOP, the foot progression angle under running is smaller than that under walking; in FFP and FFCP, the foot progression angle under running is larger than that under walking. 


\section{REFERENCES}

[1] Saunders, P.U., Pyne, D.B., Telford, R.D., Hawley, J.A (2004). Factors affecting running economy in trained distance runners. Sports Medicine, 34(7): 465-485. https://doi.org/10.2165/00007256-200434070-00005

[2] Fuller, J.T., Bellenger, C.R., Thewlis, D., Tsiros, M.D., Buckley, J.D. (2015). The effect of footwear on running performance and running economy in distance runners. Sports Medicine, 45(3): 411-422. https://doi.org/10.1007/s40279-014-0283-6

[3] Baur, H., Hirschmueller, A., Mueller, S., Mornieux, G., Divert, C., Belli, A., Mayer, F. (2003). Analysis of gait and responses to insoles in healthy runners and runners with achilles tendinitis. Medicine \& Science in Sports \& Exercise, 35(5): S91. https://doi.org/10.1097/00005768200305001-00502

[4] Ramanathan, A.K., Wallace, D.T., Arnold, G.P., Drew, T.S., Wang, W., Abboud, R.J. (2011). The effect of varying footwear configurations on the peroneus longus muscle function following inversion. The Foot, 21(1): 31-36. https://doi.org/10.1016/j.foot.2010.11.001

[5] Sole, C.C., Milosavljevic, S., Sole, G., Sullivan, S.J. (2014). Patterns of mediolateral asymmetry in worn footwear. Footwear Science, 6(3): 177-192. https://doi.org/10.1080/19424280.2014.913694

[6] McCaw, S.T., DeVita, P. (1995). Errors in alignment of center of pressure and foot coordinates affect predicted lower extremity torques. Journal of Biomechanics, 28(8): 985-988. https://doi.org/10.1016/0021-9290(94)00151-s

[7] Sole, G., Pataky, T., Sole, C.C., Hale, L., Milosavljevic, S. (2017). Age-related plantar centre of pressure trajectory changes during barefoot walking. Gait \& Posture, 57: 188-192. https://doi.org/10.1016/j.gaitpost.2017.06.016

[8] Li, Z., Liang, Y.Y., Wang, L., Sheng, J., Ma, S.J. (2016). Reliability and validity of center of pressure measures for balance assessment in older adults. Journal of Physical Therapy 28(4): 1364-1367. https://doi.org/10.1589/jpts.28.1364

[9] Luximon, Y., Cong, Y., Luximon, A., Zhang, M. (2015). Effects of heel base size, walking speed, and slope angle on center of pressure trajectory and plantar pressure when wearing high-heeled shoes. Human Movement Science, 41: 307-319.
[10] Mettler, A., Chinn, L., Saliba, S.A., McKeon, P.O., Hertel, J. (2015). Balance training and center-of-pressure location in participants with chronic ankle instability. Journal of Athletic Training, 50(4): 343-349. https://doi.org/10.4085/1062-6050-49.3.94

[11] De Cock, A., De Clercq, D., Willems, T., Witvrouw, E. (2005). Temporal characteristics of foot roll-over during barefoot jogging: Reference data for young adults. Gait \& Posture, 21(4): 432-439. https://doi.org/10.1016/j.gaitpost.2004.05.004

[12] Bizovska, L., Svoboda, Z., Kutilek, P., Janura, M., Gaba, A., Kovacikova, Z. (2014). Variability of centre of pressure movement during gait in young and middleaged women. Gait \& Posture, 40(3): 399-402. https://doi.org/10.1016/j.gaitpost.2014.05.065

[13] Willems, T.M., De Clercq, D., Delbaere, K., Vanderstraeten, G., De Cock, A., Witvrouw, E. (2006). A prospective study of gait related risk factors for exercise-related lower leg pain. Gait \& Posture, 23(1): 91-98. https://doi.org/10.1016/j.gaitpost.2004.12.004

[14] Willems, T., Witvrouw, E., Delbaere, K., De Cock, A., De Clercq, D. (2005). Relationship between gait biomechanics and inversion sprains: A prospective study of risk factors. Gait \& Posture, 21(4): 379-387. https://doi.org/10.1016/j.gaitpost.2004.04.002

[15] Allet, L., Armand, S., Golay, A., Monnin, D., De Bie, R.A., de Bruin, E.D. (2008). Gait characteristics of diabetic patients: A systematic review. Diabetes/Metabolism Research and Reviews, 24(3): 173191. https://doi.org/10.1002/dmrr.809

[16] Nolan, K.J., Yarossi, M., Mclaughlin, P. (2015). Changes in center of pressure displacement with the use of a foot drop stimulator in individuals with stroke. Clinical Biomechanics, 30(7): 755-761. https://doi.org/10.1016/j.clinbiomech.2015.03.016

[17] De Cock, A., Vanrenterghem, J., Willems, T., Witvrouw, E., De Clercq, D. (2008). The trajectory of the centre of pressure during barefoot running as a potential measure for foot function. Gait \& Posture, 27(4): 669-675. https://doi.org/10.1016/j.gaitpost.2007.08.013

[18] Gil, H., Ryu, J.S., Yoon, S., Back, K.H., Shin, J.H., Park, S.K. (2017). A new approach to quantify the centre of pressure (COP) trajectory using a shoelace formula as a potential measure of movement control during walking and running. Footwear Science, 9(S1): S86-S88. https://doi.org/10.1080/19424280.2017.1314353 